\title{
Physical activity and obesity editorial: is exercise pointless or was it a pointless exercise?
}

Kamal R Mahtani ${ }^{1}$, Jim McManus ${ }^{2}$, David Nunan ${ }^{1}$

1 - Centre for Evidence Based Medicine, Nuffield Department of Primary Care Health Sciences, University of Oxford, Oxford, UK

2 - Department of Public Health for Hertfordshire and Academy of Medical Royal Colleges

Health Inequalities Forum member, Hertfordshire County Council, Hertford, UK

Correspondence to

Dr Kamal R Mahtani, Centre for Evidence Based Medicine, Nuffield Department of Primary Care Health Sciences, University of Oxford, Radcliffe Observatory Quarter, Woodstock Road, Oxford OX2 6GG, UK; kamal.mahtani@phc.ox.ac.uk 
In their editorial, Malhotra et al 1 highlighted the important health benefits of physical activity in cardiovascular disease, type 2 diabetes, dementia and cancer, but dismissed any benefit for weight loss in obesity. However, the poor use of existing evidence to underpin this dismissal and the resultant press release generated headlines that could send a misleading message to patients and the public.

\section{Poor use of evidence}

An evidence-based approach to clinical decision-making involves understanding the best type of evidence to use, how to appraise it, and assessing its usefulness in practice. Such an approach determines the risk of bias and ensures decisions are guided by accurate, meaningful evidence. The GRADE guidelines cite expert opinion alone as very low quality evidence, with a high risk of bias.2 Unfortunately, much of the evidence cited by Malhotra and colleagues was based on expert opinion or was not referenced. For example, when excluding a relationship between obesity and physical activity, the evidence cited was an opinion piece by Luke and Cooper,3 which has been criticised for ignoring observational and experimental studies that support a relation between physical activity and obesity. They give no reference for their claim that, according to the Lancet Global Burden of Disease report, poor diet now generates more disease than physical inactivity, alcohol and smoking combined.

\section{Current recommendations for physical activity in obese patients}

To substantiate their dismissal, Malhotra and colleagues would have to provide better evidence to justify why current guidance is wrong. The WHO currently recommends that obese and overweight individuals engage in regular physical activity $(60 \mathrm{~min}$ a day for children and 150 min per week for adults).4 National Institute for Health and Care Excellence (UK) guidance advises health professionals should support obese patients in increasing their physical activity, particularly through activities that fit easily into their everyday life.5 Recognising the value of physical activity in promoting a healthy lifestyle, including in obese patients, the Academy of Medical Royal Colleges recently issued its report, Exercise: the Miracle Cure, which summarised the evidence on physical activity and called on doctors "to promote the benefits of regular physical activity to their patients and to communities in their wider roles as 'advocates for health'."6 They also highlight an overview of systematic review evidence that body weight may stay the same despite a reduction in high risk visceral fat as a result of increased muscle mass.

\section{Current evidence for physical activity and obesity}

The Malhotra editorial challenges the effectiveness of physical activity for weight loss in obese individuals. The guidelines and reports highlighted above present much of the evidence base and include a specific Cochrane systematic review by Shaw et al,7 which was ignored by the Malhotra editorial. This review included 43 studies reporting results from 41 randomised controlled trials including a total of 3476 participants. The duration of included studies ranged 
from 3 to 12 months (61\% less than 4 months), including follow-up. Although methodological quality for included studies was variable according a risk of bias assessment, the Cochrane review authors report that exercise combined with diet resulted in greater weight reduction than diet alone (mean difference $-1.0 \mathrm{~kg} ; 95 \% \mathrm{Cl},-1.3$ to -0.7 ). They also note the finding that increasing exercise intensity increased the magnitude of weight loss (mean difference $-1.5 \mathrm{~kg}$; $95 \% \mathrm{Cl}-2.3$ to -0.7$)$. An additional finding was that exercise improved a range of secondary outcomes even when weight loss did not occur. The authors conclude exercise was an effective weight loss intervention, particularly when combined with dietary interventions, but make no inference to the poor quality of included trials and little reference to the fact that diet alone appeared better than exercise alone (mean difference $-3.6 \mathrm{~kg}, 95 \% \mathrm{Cl}-4.3$ to -3 ). These outcome and quality findings are repeated in a more recent systematic review assessing treatment (including diet, exercise or diet and exercise) for overweight and obesity in adult populations. 8 Whether these differences are meaningful requires good clinical judgement and open, honest discussion. However, when objectively assessing the best available evidence, for which we acknowledge the majority is of low quality, it would appear that diet alone (mainly low fat) is better than exercise alone but better than either are diet and exercise together. A further key point is that improvements in important health risk factors are observed with exercise even without weight loss.

\section{Conclusions}

There is little doubt that diet is key in the management of the obesity epidemic. Indeed, Malhotra and colleagues could be congratulated for taking on the 'food industry's public relations machinery' and they helpfully point out that more care is needed in relationships between sport and celebratory endorsements of junk food or sports drinks. What is less helpful, and does no one, including the public and patients, any favours, is the production of opinion pieces that use evidence poorly or not at all.

\section{Acknowledgments}

The authors would like to thank Jeffrey Aronson and Meena Mahtani for editorial comments.

Competing interests KRM is an NHS general practitioner who supports and advises patients to lose weight through a healthy balanced diet and regular physical activity. He holds NHS National Institute of Health Research (NIHR) grant funding for some of his research. JMcM is Director of Public Health for Hertfordshire, and a member of the Academy of Medical Royal Colleges Health Inequalities Forum. He is a member of the International Scientific Advisory Group for the Centre for Diet and Activity Research at the University of Cambridge. DN is a senior research fellow and tutor of evidence-based medicine, with a background and research interest in exercise physiology and physical activity. He holds NHS National Institute of Health Research (NIHR) grant funding for some of his research. The views expressed are those of the author(s) and not necessarily those of the NHS National Institute of Health Research (NIHR) or employing institutions. 


\section{References}

1 Malhotra A, Noakes T, Phinney S. It is time to bust the myth of physical inactivity and obesity: you cannot outrun a bad diet. Br J Sports Med 2015;49: 967-8.

2 Balshem $\mathrm{H}$, Helfand $\mathrm{M}$, Holger J, et al. GRADE guidelines: 3. Rating the quality of evidence. J Clin Epidemiol 2011;64: 401-6.

3 Luke A, Cooper RS. Physical activity does not influence obesity risk: time to clarify the public health message. Int J Epidemiol 2013;42:1831-6.

4 http://www.who.int/mediacentre/factsheets/fs311/en/ (accessed 28 Apr 2015).

5 http://www.nice.org.uk/guidance/cg43/resources/ guidance-obesity-pdf (accessed 28 Apr 2015).

6 http://www.aomrc.org.uk/general-news/exercise-themiracle-cure.html (accessed 28 Apr 2015).

7 Shaw KA, Gennat HC, O'Rourke P, et al. Exercise for overweight or obesity. Cochrane Database Syst Rev 2006;(4):CD003817.

8 Peirson L, Douketis J, Ciliska S, et al. Treatment for overweight and obesity in adult populations: a systematic review and meta-analysis. CMAJ Open 2014;2:E306-17. 